\title{
Psychological experimentation through direct connection of the subject interface to a VAX
}

\author{
PETER G. POLSON, JAMES R. MILLER, and JOHN KARAT \\ Department of Psychology and Computer Laboratory for Instruction in Psychological Research \\ University of Colorado, Boulder, Colorado 80309
}

\begin{abstract}
We outline the rationale for using a DEC VAX-11/780 for the real-time control of psychological experiments. The properties of VAX hardware and software that make the system well suited to the requirements of many classes of psychological experiments, particularly those that require the concurrent control of several independent experiments or the control of one experiment that must be written as several intercommunicating processes are discussed.
\end{abstract}

During the last year and a half, several psychology departments have acquired or have planned to acquire Digital Equipment Corporation (DEC) VAX 11/780 computers as central computing facilities for their organizations. Most groups are using or plan to use the VAX for data processing, word processing, cognitive simulation, and other typical timesharing activities. In addition, the VAX can play two roles in the execution of psychological experiments: as a central machine storing programs, stimuli, and data files manipulated by smaller laboratory computers networked to the VAX, and as the system actually in control of experimental stations. The purpose of this paper is to outline why we intend to use our VAX for experimental control, in addition to the other activities noted above.

The Computer Laboratory for Instruction in Psychological Research (CLIPR) is a central computing facility that provides real-time and general interactive computing for a number of the laboratories in the psychology department at the University of Colorado (Bailey, 1972; Bailey \& Polson, 1975; Polson, 1978). CLIPR was originally built around a single central machine, the Xerox Sigma 3. Centralized computing resources permit one to acquire a system with more power and peripherals than would be available to a single individual. A larger machine provides a more congenial environment for software development, with a wider range of software tools, and the efforts of the installation's staff toward such development will typically benefit all the research programs using the facility. Finally, the same software used to support various research programs can also support a department's instructional program. Such arrangements are often difficult, if not impossible, if each laboratory has independent facilities that are often the private resources of a single individual.

This summer, we replaced the Sigma 3 with a VAX running the VMS operating system. Careful study con-

Requests for reprints should be sent to Peter G. Polson, Department of Psychology, University of Colorado, Boulder, Colorado 80309. The Computer Laboratory for Instruction in Psychological Research is supported in part by funds from the University of Colorado. vinced us that the VAX would satisfy existing real-time computing needs and, at the same time, provide much needed expansion of facilities relevant to interactive program development, data analysis, and cognitive simulation. The VAX will provide general interactive computing for our department and will serve as a host to several PDP 11/03 systems via DECNET, and its realtime facilities will directly support several research programs.

One of the problems faced by psychological computing facilities is the diversity of needs posed by the variety of experimental paradigms within psychology. There are many simple paradigms that can be satisfied by inexpensive microprocessors. Other domains have such high-density processing requirements, such as eye movement monitoring, that a dedicated processor is generally required. There is an intermediate set of paradigms, however, that can be characterized by relatively infrequent responses, the accurate timing of these responses, and a nontrivial amount of computation between the responses. Experiments in human problem solving provide an example of this type of task.

Jeffries and Karat (1979) have described a system for controlling experiments utilizing various puzzle problems, such as river-crossing and Tower of Hanoi tasks. This system must record the subject's responses, evaluate the legality of the subject's move, and determine the proper display to be presented to the subject following his move. The data structures and programming required here would likely exceed the capacity of small microprocessors, and they might be best run as independent, but intercommunicating, concurrent processes that assume the existence of an efficient multitask/ multiprogramming operating system on the controlling computer. Such an operating system may not be avail. able on small computers.

Progress in the simulation of cognitive processes also calls for more complex experimental control systems. As the sophistication of cognitive simulation models increases, experiments in which the selection or generation of stimuli and interpretation of the subject's responses is determined by the simulation model itself 
becomes increasingly feasible. Such a paradigm would almost certainly require that the experimental system and the simulation model run as concurrent processes. In addition, the language requirements of simulation modeling may restrict the types of machines that can control these experiments. The value of the VAX for experimentation in psychology is that it meets the requirements of these paradigms. We will outline some of the features that are of value in meeting the requirements described above.

\section{FAST CONTEXT SWITCH}

Critical to the success of any multiprogrammed realtime system is the ability to switch rapidly from one task to another on the occurrence of an internal or external interrupt. The Sigma 3 could do a full context switch in less than 100 microsec; the VAX takes approximately 180 microsec. In contrast, it has been reported to us that a PDP-11 running RSX-11M can take up to 1,000 microsec for a full context switch. This is clearly insufficient for the needs of most experiments.

\section{MULTILEVEL PRIORITY INTERRUPT SYSTEM}

Experience with the Sigma 3 led us to the conclusion that real-time computing resources are best allocated by a multilevel priority interrupt system in which a given task runs to completion unless it is interrupted by the demands of a higher priority task. At the top of such a priority chain are tasks that control the duration of time-critical events and that record response latencies. Tasks lower in the priority chain carry out various experiment-related computations, generate stimuli, and store the data collected by these higher priority tasks.

To meet these needs, the VAX has 32 levels of priority. The lower 16 levels are used by the timesharing system to distribute resources among active processes to optimize the total efficiency of the system. Jobs that have small computational requirements but occasional bursts of response-oriented $\mathrm{I} / \mathrm{O}$ are temporarily increased in priority in order to maintain the total responsiveness of the system. Thus, the system scheduler allocates and manipulates the priorities of tasks running at the lower 16 levels of priority. Jobs sharing a common level of priority are run on a round-robin basis.

The VAX can be used as a real-time system by assigning to a process one of the upper 16 priority levels. These upper levels take priority over all other tasks in the machine, including the scheduler and the swapper. In addition, these priorities can be shifted during the execution of a program, so that only the time-critical parts of the program need run at a particularly high priority. The only tasks that take precedence over all user processes are certain error-recovery procedures that must execute to preserve the integrity of the total system. The important point here is that, except for hardware requirements, there are no programming differences between real-time and low priority processes.
Programs that will ultimately run as real-time experimental control processes can be developed in the normal timesharing environment, tested at standard priority levels, and assigned a real-time priority level upon completion.

\section{MEMORY}

A large number of real-time tasks can be effectively executed simultaneously only if at least some part of the tasks' programs are resident in memory. The Sigma 3 had no provisions for automatically swapping programs in and out of memory. Hence, because of the limited address space of the Sigma 3, complex user-specified overlay techniques were required to allow programs of reasonable size and to insure that the time-critical parts of these programs were always resident in memory. The VAX has no such problems. First, the VAX supports up to 8 million bytes of physical memory, so one can keep in memory a very large number of real-time programs for immediate execution. Second, the VMS operating system in which only a small portion of a program is memory resident. When nonresident segments (pages) of the program are required, they are automatically brought into core by the pager; unneeded pages are swapped back to the disk. The important feature is that this paging is under program control. A user can lock time-critical segments of the program into memory and allow less critical pages to be swapped to and from the disk. This control over the virtual memory system, coupled with the ability to manipulate interrupt levels, permits the programmer to make very sophisticated use of the VAX's resources.

\section{SYSTEM SERVICES AND RESOURCE ALLOCATION}

A significant portion of our development of the Sigma 3 was devoted to writing FORTRAN interfaces to the various system services that were needed by real-time programmers. One of the strengths of the VAX is that VMS is a completely modular operating system, with all elements of the operating system accessible to the programmer in high-level languages. For example, the system's command language scanner, the module responsible for interpreting an interactive user's commands, can be accessed from FORTRAN, with the result that a FORTRAN program can do anything that can be done by an interactive user.

This flexibility would be worthless without a sound system for resource allocation, control, and protection. VMS meets these requirements in several ways. Various peripheral devices and specialized hardware interfaced to the system can be allocated under control of the operating system for the exclusive use of a given program. The operating system provides relatively complete protection from interference or inadvertant modification of one program by another.

VMS offers almost complete control of an individual 
user's access to the system's more advanced facilities Each of these facilities of the system can be granted or denied to an individual user. This makes the VAX an ideal system for an instructional and research environment. Those users that have the ability to use sophisticated system facilities or to manipulate system parameters can be given permission to do so on a case-by-case basis. In addition, privileges can be given to processes as well as to users: A data collection subroutine can be written that, in order to assure accurate response timing, raises its priority during the actual collection of the response. The system privilege of priority alteration can be assigned to this subroutine, rather than to the user of the routine.

\section{THE USER INTERFACE}

There is a reasonably limited number of basic commands on the VAX, and these commands default to very reasonable modes of operation. For example, the command to cause the linking loader to generate an executable image requires that a naive user specify only the name of the file containing the object code to be linked. However, there are over 30 parameters and options that the sophisticated user can specify to control the operation of the linker. This access to the components of the operating system permits the sophisticated user to write very complex and powerful real-time programs.

Our previous discussion is focused on those features of the VAX that we think make it an ideal machine for real-time computing. The machine has a full set of primitives, including event flags, message passing between processes, the ability to create shareable code, and all of the facilities needed to write complex multitask real-time programs. As such, it is the most sophisticated and easily used real-time system that any of us at CLIPR have dealt with. We are currently developing new software and converting old software from the Sigma 3 to determine how these aspects of the VAX architecture are realized in a real-world system.

\section{REFERENCES}

BAItey, D. E. The computer laboratory for instruction in psychological research: CLIPR. Behavior Research Methods \& Instrumentation, 1972, 4, 95-96.

Bailey, D. E., \& Polson, P. G. Real-time computing at the University of Colorado. American Psychologist, 1975, 30, 212-218.

Jeffries, R., \& KarAT, J. A general-purpose program for problem solving experimentation. Behavior Research Methods \& Instrumentation, 1979, 11, 205-207.

Polson, P. G. Microprocessors: Their impact on real-time computing in psychology. Behavior Research Methods \& Instrumentation, 1978, 10, 139-147. 